\title{
A mathematical model for assembly line balancing model to consider disordering sequence of workstations
}

\author{
William Ho,* and Ali Emrouznejad \\ Operations and Information Management Group \\ Aston Business School, Aston University \\ Birmingham B4 7ET, United Kingdom
}

\begin{abstract}
Patterson and Albracht [1] formulated a binary integer programming model for the simple assembly line balancing problem, which is well known as SALBP-1, in more than 30 years ago. Since then, a number of researchers have extended the model for the variants of assembly line balancing problem. The model is still prevalent nowadays mainly because of the lower and upper bounds on task assignment. These properties avoid significant increase of decision variables. In this paper we use an example to show that the model may lead to a confusing solution. We then provide a "remedial" constraint set for the model to rectify the disordered sequence problem.
\end{abstract}

Keywords: Assembly line balancing; Single model; mathematical programming; SALBP-1

\footnotetext{
*Corresponding author: Dr William Who, Operations and Information Management Group, Aston Business School, Aston University, Birmingham B4 7ET, United Kingdom,

Email: w.ho@aston.ac.uk, Tel: +44 (0)121 2043342.
} 


\section{Introduction}

Assembly lines are flow-oriented production systems which are mainly designed for continuous production of high-volume and low-variety commodities. An assembly line consists of workstations arranged along a conveyor belt or a similar mechanical material handling equipment. The commodities are consecutively passed through the line and are moved from a workstation to the next. At each workstation, several tasks are repeatedly performed and their operation sequence or precedence relationship is predetermined. A simple assembly line balancing problem (SALBP) can be stated as follows. Given a set of tasks, time requirement of each task, and precedence relationship of tasks, the problem is to assign the tasks to an ordered sequence of workstations while meeting the production requirement at minimum number of workstations (SALBP-1) or minimum cycle time (SALBP-2).

The SALBP and its variants have been studied extensively, for which a number of mathematical modelling techniques (e.g., binary integer programming and goal programming), exact algorithms (e.g., branch-and-bound algorithm and dynamic programming approaches), and heuristic approaches (e.g., genetic algorithm, tabu search, and ant colony optimization approach) have been developed [2]. Among the mathematical modelling techniques, the binary integer programming model developed by Patterson and Albracht [1] is one of the most widely studied formulations for the SALBP-1. The model is still prevalent nowadays mainly because of the lower and upper bounds on task assignment. By defining the earliest and latest workstations to which a task can be assigned, the number of binary decision variables can be significantly reduced. Using the concept of SALBP-1 model, Gökçen and Erel [3, 4] developed a binary goal programming model and a binary integer programming model for the mixed-model assembly line balancing problem. Further, Ağpak and Gökçen [5] used SALBP-1 model to develop a binary integer programming model for the resource constrained assembly line balancing problem, while Amen [6] proposed a cost-oriented assembly line balancing problem with wages and capital cost which is a generalization of SALBP-1 and Scholl and Becker [7] drawn an exact method for cost-oriented assembly line balancing based on SALBP-1. More recently, Andres et al [8] added sequence-dependent setup time considerations to the classical SALBP model and 
Toksari et al [9] extended the classical SALBP to include learning effect into assembly line balancing problems. A state-of-the-art exact and heuristic solution procedure for simple assembly line balancing is developed by Scholl and Becker [10].

In this paper we use an example to show that the SALBP-1 model may lead to a confusing solution. We then provide a "remedial" constraint set for the model to rectify the disordered sequence problem.

This paper is organized as follows. Section 2 presents the standard SALBP-1 model, and the lower and upper bounds on task assignment. Section 3 uses a numerical example to demonstrate that the model leads to a confusing solution, and then provides a "remedial" constraint set for the model. Finally, Section 4 concludes the paper.

\section{SALBP-1 model}

Patterson and Albracht [1] formulated a binary integer programming model for the SALBP with minimum number of workstations, that is, SALBP-1. Consider a work with a series of tasks $i=\{1,2, \ldots, m\}$ to be assigned to a number of workstations $j=\{1$, $2, \ldots, n$ \}. The objective is to minimize the number of workstations, $A_{j}$, used to complete the entire work. Given the precedence relationships, the earliest workstation to which task $i$ can be assigned is denoted as $E_{i}$, whereas the latest workstation to which task $i$ can be assigned is denoted as $L_{i}$. The time required by task $i$ is denoted as $t_{i}$, whereas the theoretical cycle time is denoted as $C$. $W_{j}$ refers to the subset of all tasks that can be assigned to workstation $j$, whereas $\left\|W_{j}\right\|$ refers to the number of tasks in subset $W_{j}$. $P_{i}$ refers to the set of tasks that must proceed task $i$, whereas $S_{i}$ refers to the set of tasks that must succeed task $i$. By defining decision variables $x_{i j}$ to represent the assignment of task $i$ to workstation $j$, the Patterson and Albracht's (1975) model for the SALBP-1 can be written as

$$
\text { Minimize } z=\sum_{j=1}^{n} A_{j}
$$

subject to

$$
\begin{array}{ll}
\sum_{j=E_{i}}^{L_{i}} x_{i j}=1 & i=1,2, \ldots, m \\
\sum_{i \in W_{j}} t_{i} x_{i j} \leq C & j=1,2, \ldots, n
\end{array}
$$




$$
\begin{array}{ll}
\sum_{j=E_{a}}^{L_{a}} j x_{a j} \leq \sum_{j=E_{b}}^{L_{b}} j x_{b j} & \text { for } \forall(a, b) \in P \\
\sum_{i \in W_{j}} x_{i j} \leq\left\|W_{j}\right\| A_{j} & j=1,2, \ldots, n
\end{array}
$$

All $x_{i j}$ and $A_{j}=0$ or 1 .

where

$$
\begin{aligned}
& E_{i}=\left[\left(t_{i}+\sum_{k \in P_{i}} t_{k}\right) / C\right]^{+} \\
& L_{i}=n+1-\left[\left(t_{i}+\sum_{k \in S_{i}} t_{k}\right) / C\right]^{+}
\end{aligned}
$$

The objective function (1) is to minimize the number of workstations used to complete the entire work. Constraint set (2) is an assignment constraint, which guarantees that each task is assigned to exactly one workstation. Constraint set (3) is a cycle time constraint, which guarantees that the sum of task times assigned to each workstation does not exceed the theoretical cycle time (i.e., maximum time allowed at each workstation). Constraint set (4) is a precedence relationship constraint, which ensures that task $b$ cannot be performed until task $a$ is finished, where task $a$ is an immediate predecessor of task $b$. Constraint set (5) is a workstation constraint, which ensures that a workstation is utilized if the task(s) is/are assigned to it.

Equation (6) computes the lower bound or the earliest workstation to which task $i$ can be assigned. Equation (7) computes the upper bound or the latest workstation to which task $i$ can be assigned. In equations (6) and (7), $[y]^{+}$denotes the smallest integer greater than or equal to $y$.

\section{Disordered sequence of workstations}

This section shows that the solution to the SALBP-1 model may be disordered, which means that the workstations to which the tasks are assigned may not be in an ordered sequence. To illustrate this, we apply a numerical example with eight tasks. The precedence relationship of tasks, time requirement of each task, lower and upper bounds of task assignment are shown in Table 1 . The theoretical cycle time is given as 3.7 
minutes. Note that the maximum number of workstations available is five. The model for this example consists of 31 binary decision variables and 27 constraints.

Table 1. Precedence relationship, $\underline{t}_{i}, E_{i}$ and $L_{i}$ of a work

\begin{tabular}{|c|c|c|c|c|}
\hline Task, $i$ & Immediate predecessors & $t_{i}$ & $E_{i}$ & $L_{i}$ \\
\hline 1 & - & 1.0 & 1 & 2 \\
\hline 2 & 1 & 0.6 & 1 & 3 \\
\hline 3 & 1 & 1.6 & 1 & 5 \\
\hline 4 & 2 & 2.7 & 2 & 4 \\
\hline 5 & 2 & 1.2 & 1 & 4 \\
\hline 6 & 4 & 0.9 & 2 & 5 \\
\hline 7 & 5 & 3.4 & 2 & 4 \\
\hline 8 & $3,6,7$ & 1.1 & 4 & 5 \\
\hline
\end{tabular}

The optimal solution to this example is yielded as: $x_{11}=x_{21}=x_{35}=x_{43}=x_{51}=x_{63}$ $=x_{74}=x_{85}=1$, which implies that $A_{1}=\{1,2,5\}, A_{3}=\{4,6\}, A_{4}=\{7\}$, and $A_{5}=\{3,8\}$. The optimal solution value is four $\left(A_{1}=A_{3}=A_{4}=A_{5}=1\right)$. Obviously, workstation 2 is omitted, and this is regarded as disordered sequence of workstations. To prevent from generating this kind of confusing solution, an addition constraint set must be incorporated into the model as follows.

$$
A_{j} \geq A_{k} \quad \text { for } \forall(j, k) \in n ; k>j
$$

Obviously constraint set (8) ensures that an earlier workstation is utilized if the task(s) is/are assigned to its following workstation(s). This is because the optimum solution of SALBP problem must obtain only if $A_{j} \geq A_{k}$ (for all $k>j$ ) hence this constraint set prevents of any diordering sequence of worksations. Next section demonstrates an example to show how the extra constraint set (8) affects the results for SALBP model in Table 1. This is the smallest example of this kind that the authors found.

\section{Modified SALBP-1 model}

The modified SALBP-1 model for the example given in table (1) is as follows. 
Minimize $z=A_{1}+A_{2}+A_{3}+A_{4}+A_{5}$

subject to

$$
\begin{aligned}
& x_{11}+x_{12}=1 \\
& x_{21}+x_{22}+x_{23}=1 \\
& x_{31}+x_{32}+x_{33}+x_{34}+x_{35}=1 \\
& x_{42}+x_{43}+x_{44}=1 \\
& x_{51}+x_{52}+x_{53}+x_{54}=1 \\
& x_{62}+x_{63}+x_{64}+x_{65}=1 \\
& x_{72}+x_{73}+x_{74}=1 \\
& x_{84}+x_{85}=1 \\
& x_{11}+0.6 x_{21}+1.6 x_{31}+1.2 x_{51} \leq 3.7 \\
& x_{12}+0.6 x_{22}+1.6 x_{32}+2.7 x_{42}+1.2 x_{52}+0.9 x_{62}+3.4 x_{72} \leq 3.7 \\
& 0.6 x_{23}+1.6 x_{33}+2.7 x_{43}+1.2 x_{53}+0.9 x_{63}+3.4 x_{73} \leq 3.7 \\
& 1.6 x_{34}+2.7 x_{44}+1.2 x_{54}+0.9 x_{64}+3.4 x_{74}+1.1 x_{84} \leq 3.7 \\
& 1.6 x_{35}+0.9 x_{65}+1.1 x_{85} \leq 3.7 \\
& x_{11}+2 x_{12}-x_{21}-2 x_{22}-3 x_{23} \leq 0 \\
& x_{11}+2 x_{12}-x_{31}-2 x_{32}-3 x_{33}-4 x_{34}-5 x_{35} \leq 0 \\
& x_{21}+2 x_{22}+3 x_{23}-2 x_{42}-3 x_{43}-4 x_{44} \leq 0 \\
& x_{21}+2 x_{22}+3 x_{23}-x_{51}-2 x_{52}-3 x_{53}-4 x_{54} \leq 0 \\
& x_{31}+2 x_{32}+3 x_{33}+4 x_{34}+5 x_{35}-4 x_{84}-5 x_{85} \leq 0 \\
& 2 x_{42}+3 x_{43}+4 x_{44}-2 x_{62}-3 x_{63}-4 x_{64}-5 x_{65} \leq 0 \\
& x_{51}+2 x_{52}+3 x_{53}+4 x_{54}-2 x_{72}-3 x_{73}-4 x_{74} \leq 0 \\
& 2 x_{62}+3 x_{63}+4 x_{64}+5 x_{65}-4 x_{84}-5 x_{85} \leq 0 \\
& 2 x_{72}+3 x_{73}+4 x_{74}-4 x_{84}-5 x_{85} \leq 0 \\
& x_{11}+x_{21}+x_{31}+x_{51}-4 A_{1} \leq 0 \\
& x_{12}+x_{22}+x_{32}+x_{42}+x_{52}+x_{62}+x_{72}-7 A_{2} \leq 0 \\
& x_{23}+x_{33}+x_{43}+x_{53}+x_{63}+x_{73}-6 A_{3} \leq 0 \\
& x_{34}+x_{44}+x_{54}+x_{64}+x_{74}+x_{84}-6 A_{4} \leq 0 \\
& x_{35}+x_{65}+x_{85}-3 A_{5} \leq 0 \\
& A_{1}-A_{2} \geq 0 \\
& A_{1}-A_{3} \geq 0 \\
& A_{1}-A_{4} \geq 0
\end{aligned}
$$




$$
\begin{aligned}
& A_{1}-A_{5} \geq 0 \\
& A_{2}-A_{3} \geq 0 \\
& A_{2}-A_{4} \geq 0 \\
& A_{2}-A_{5} \geq 0 \\
& A_{3}-A_{4} \geq 0 \\
& A_{3}-A_{5} \geq 0 \\
& A_{4}-A_{5} \geq 0 \\
& \text { All } x_{i j} \text { and } A_{j}=0 \text { or } 1 .
\end{aligned}
$$

The optimal solution to this example is yielded as: $x_{11}=x_{21}=x_{34}=x_{42}=x_{51}=x_{64}=x_{73}=$ $x_{84}=1$, which implies that $A_{1}=\{1,2,5\}, A_{2}=\{4\}, A_{3}=\{7\}$, and $A_{4}=\{3,6,8\}$. The optimal solution value is four $\left(A_{1}=A_{2}=A_{3}=A_{4}=1\right)$.

\section{Conclusions}

This paper showed that the mathematical model of SALBP-1 as developed by Patterson and Albracht [1], and prevalently adopted by various researchers may produce inappropriate results. The solution generated may have disordered sequence of workstations. An additional constraint set was, therefore, proposed and incorporated into the model to ensure that the tasks are always assigned to an ordered sequence of workstations.

More recently, the concept of the assembly line balancing research has been extended towards formulating generalized assembly balancing problems (GALBP) with extra characteristics such as cost functions, equipment selection, and U-shaped line layout [10]. Thus, additional research is necessary to investigate similar disordering sequence to the variety of GALBP.

\section{Reference}

[1] Patterson, J. H., Albracht, J. J., 1975. Assembly line balancing: zero-one programming with Fibonacci search. Operations Research 23, 166-172.

[2] Scholl, A., Becker, C., 2006. State-of-the-art exact and heuristic solution procedures for simple assembly line balancing. European Journal of Operational Research 168, 666-693. 
[3] Gökçen, H., Erel, E., 1997. A goal programming approach to mixed-model assembly line balancing problem. International Journal of Production Economics 48, 177-185.

[4] Gökçen, H., Erel, E., 1998. Binary integer formulation for mixed-model assembly line balancing problem. Computers and Industrial Engineering 34, 451-461.

[5] Ağpak, K., Gökçen, H., 2005. Assembly line balancing: two resource constrained cases. International Journal of Production Economics 96, 129-140.

[6] Amen, M., 2000. An exact method for cost-oriented assembly line balancing. International Journal of Production Economics 64, 187-195.

[7] Scholl, A., Becker, C., 2005. A note on 'An exact method for cost-oriented assembly line balancing', International Journal of Production Economics 97 (2005) 343-352.

[8] Andres, C. Miralles, C., Pastor, R., 2008. Balancing and scheduling tasks in assembly lines with sequence-dependent setup times, European Journal of Operational Research 187(3) 1212-1223.

[9] Toksari, M. D., Isleyen, S. K., Guner, E., Baykoc, O. F., 2008. Simple and U-type assembly line balancing problems with a learning effect, Applied Mathematical Modelling, In Press.

[10] Becker, C., Scholl, A., 2006. A survey on problems and methods in generalized assembly line balancing, European Journal of Operational Research 168(3) 694-715. 


\section{University Library}

\section{- M M N E R VA A gateway to Melbourne's research publications}

Minerva Access is the Institutional Repository of The University of Melbourne

Author/s:

Ho, W;Emrouznejad, A

Title:

A mathematical model for assembly line balancing model to consider disordering sequence of workstations

Date:

2009-01-01

Citation:

Ho, W. \& Emrouznejad, A. (2009). A mathematical model for assembly line balancing model to consider disordering sequence of workstations. ASSEMBLY AUTOMATION, 29 (1), pp.49-51. https://doi.org/10.1108/01445150910929857.

Persistent Link:

http://hdl.handle.net/11343/118669 